\title{
EFEITOS ANESTÉSICOS DA ADMINISTRAÇÃO INTRANASAL OU INTRAMUSCULAR DA ASSOCIAÇÃO DE MIDAZOLAM E CETAMINA RACÊMICA OU S+ EM PERIQUITO AUSTRALIANO (Melopsittacus undulatus)
}

\author{
ANESTHETICS EFFECTS OF INTRANASAL OR INTRAMUSCULAR \\ ASSOCIATION OF MIDAZOLAM AND RACEMIC OR S+ KETAMINE IN \\ BUDGERIGARS (Melopsittacus undulatus)
}

\author{
Gustavo Aléssio Trevisan ${ }^{1}$ \\ Everton Leonardi da Silva ${ }^{1}$ \\ Anderson Luiz de Carvalho ${ }^{1}$ \\ Rafael Messias Luiz ${ }^{*}$
}

1 Universidade Federal do Paraná, Palotina, PR, Brasil

*Autor para correspondência - rafaelmessiasluiz@gmail.com

\begin{abstract}
Resumo
A anestesia intranasal em aves é considerada uma técnica anestésica segura, simples e eficiente. O objetivo deste estudo foi comparar os efeitos anestésicos da associação de midazolam $\left(5 \mathrm{mg} \cdot \mathrm{kg}^{-1}\right) \mathrm{e}$ cetamina $\left(15 \mathrm{mg} \cdot \mathrm{kg}^{-1}\right)$ nas formulações racêmica $(\mathrm{R})$ ou $\mathrm{S}+(\mathrm{S})$ administrados pela via intranasal (IN) ou intramuscular (IM) em periquitos australianos (Melopsittacus undulatus). Foram utilizados oito periquitos em delineamento do tipo crossover, em quatro tratamentos: INR, INS, IMR e IMS. Foram avaliados os tempos de latência, decúbito dorsal, anestesia e recuperação, grau de sedação e qualidade de recuperação. Foi observada diferença significativa no tempo de latência entre INS $(40,25 \pm 10,55$ seg) e IMR $(74,32 \pm 21,77 \mathrm{seg})$; entre as vias de administração para o tempo de decúbito dorsal, INS $(23,93 \pm 7,51 \mathrm{~min})$ e INR $(28,68 \pm 16,13 \mathrm{~min})$, diferente de IMS $(60,08 \pm 27,37 \mathrm{~min})$ e IMR $(74,3 \pm 21,77$ $\mathrm{min})$ e para tempo de anestesia, INS (45,48 $\pm 17,94 \mathrm{~min})$ e INR $(39,24 \pm 15,62 \mathrm{~min})$, diferentes de IMS $(75,84 \pm 20,20 \mathrm{~min})$ e IMR $(79,4 \pm 20,73 \mathrm{~min})$. O tempo de recuperação foi significativamente maior em INS (21,55 $\pm 18,43 \mathrm{~min})$ comparado a IMR (5,1 $\pm 3,56 \mathrm{~min})$. Pode-se concluir que as duas vias de administração avaliadas podem ser utilizadas em procedimentos de curta duração e não invasivos e a via intranasal é preferível para procedimentos rápidos.
\end{abstract}

Palavras-chave: aves; benzodiazepínico; dissociativa; contenção química; anestesia intranasal.

\begin{abstract}
Intranasal anesthesia in birds is considered a safe, simple and efficient technique. The aim of this study was compare the anesthetic effects of midazolam $\left(5 \mathrm{mg} \cdot \mathrm{kg}^{-1}\right)$ with racemic $(\mathrm{R})$ or $\mathrm{S}+(\mathrm{S})$ ketamine (15 mg.kg-1 ${ }^{-1}$, administered intranasally (IN) or intramuscularly (IM) in budgerigars (Melopsittacus undulatus). Eight budgerigars were used in a crossover design with four treatments: INR, INS, IMR and IMS. Onset time, dorsal recumbency time duration, total anesthesia time, total recovery time, sedation degree and recovery quality were evaluated. Significant differences were observed in onset
\end{abstract}


time between INS $(40.25 \pm 10.55 \mathrm{sec})$ and IMR $(74.32 \pm 21.77 \mathrm{sec})$; between administration vials for dorsal recumbency time, INS $(23.93 \pm 7.51 \mathrm{~min})$ and INR $(28.68 \pm 16.13 \mathrm{~min})$, which were different from IMS $(60.08 \pm 27.37 \mathrm{~min})$ and IMR $(74.3 \pm 21 \mathrm{~min})$. In total anesthesia time, INS $(45.48 \pm 17.94$ $\mathrm{min})$ and INR $(39.24 \pm 15.62 \mathrm{~min})$ were different from IMS $(75.84 \pm 20.20 \mathrm{~min})$ and IMR $(20.73 \pm 79.4$ $\mathrm{min})$. The total recovery time was significantly higher in INS $(21.55 \pm 18.43 \mathrm{~min})$ compared to IMR $(5.1 \pm 3.56 \mathrm{~min})$. The results of this study indicated that both administration vials can be used for short time non-invasive procedures and the intranasal vial is preferable for fast procedures.

Keywords: benzodiazepinics; birds; chemical restraint; dissociative; intranasal anesthesia.

Enviado em: 28 julho 2014

Aceito em: 17 novembro 2015

\section{Introdução}

A procura por pets exóticos, em especial as aves, cria para o médico veterinário, que é requisitado para a realização de procedimentos clínicos e cirúrgicos, um novo nicho de trabalho ${ }^{(1)}$. No entanto, o manejo das aves é uma atividade que demanda extremo cuidado do médico veterinário, pois são espécies de pequeno porte, delicadas e que necessitam de manejo cauteloso ${ }^{(2,3)}$.

As aves reagem de maneira desfavorável à manipulação ${ }^{(3)}$, sendo necessária contenção física para exames e procedimentos, situações que geram estresse e acarretam em liberação de catecolaminas, arritmias, hipercapnia e podem levar ao óbito ${ }^{(2,4)}$. Para minimizar o estresse e os riscos da manipulação, é recomendado o emprego de sedação e anestesia geral ${ }^{(5-7)}$, o que pode ser um desafio para a maioria dos médicos veterinários ${ }^{(8)}$.

A anestesia inalatória é a técnica anestésica mais indicada para aves ${ }^{(9)}$, tendo como vantagens rápida indução e recuperação anestésica com mínimos efeitos cardiovasculares e de biotransformação hepática ${ }^{(8)}$. Mesmo sendo a técnica mais indicada, a anestesia inalatória não é recomendada nos casos de fraturas em ossos pneumáticos, rupturas de sacos aéreos e procedimentos em trato respiratório ${ }^{(10)}$, devendo, nesses casos, se empregar a anestesia injetável.

Como técnica alternativa à anestesia inalatória, a anestesia injetável é indicada para condições a campo e de indisponibilidade de equipamentos de anestesia inalatória, tendo como vantagens baixo custo e praticidade $^{(5,11)}$. As desvantagens das técnicas injetáveis são o estresse, possibilidade de administração intravascular ou celomática, dor à aplicação, necessidade de biotransformação hepática, recuperação agitada e prolongada ${ }^{(1,6,8)}$.

A anestesia intranasal é uma técnica de anestesia injetável não invasiva, descrita por Machin \& Caulkett ${ }^{(12)} \mathrm{e}$ Vesal \& Zare ${ }^{(6)}$, que proporciona pouco estresse à ave devido à rápida contenção e pouca manipulação, sendo indicada para sedação e anestesia. Bigham \& Moghaddam $^{(13)}$ demonstraram que a $^{2}$ sedação intranasal proporciona sedação rápida e segura, de recuperação suave e livre de complicações. São descritas diferentes associações para anestesia pela via intranasal em aves, sendo a associação de cetamina e midazolam indicada por promover anestesia de curta duração, miorrelaxamento e recuperação rápida e calma ${ }^{(3,14,15)}$.

A cetamina é um dos principais fármacos empregados na anestesia em aves, produzindo efeitos variáveis nas diferentes espécies ${ }^{(2)}$. Entretanto, os efeitos da cetamina $\mathrm{S}+$ são pouco conhecidos nestes animais, ao passo que em mamíferos esta formulação apresenta maior potência anestésica ${ }^{(16,17)}$ e causa menos efeitos indesejáveis como catatonia e ataxia, além de promover rápida recuperação anestésica $^{(18)}$.

Os objetivos do presente estudo foram avaliar a viabilidade e comparar os efeitos anestésicos da associação de midazolam e cetamina racêmica ou cetamina $\mathrm{S}+$ administrados pela via intranasal ou intramuscular em periquitos australianos (Melopsittacus undulatus). 


\section{Material e Métodos}

O presente estudo foi aprovado pela Comissão de Ética no Uso de Animais da instituição de origem, sob o protocolo 13/2013. Foram utilizados oito periquitos australianos (Melopsittacus undulatus) adultos jovens, hígidos, de ambos os sexos, alocados em viveiro coletivo com água e ração comercial ad libitum. As aves passaram por um período de adaptação ambiental, alimentar e de manejo de quatro semanas.

Após o período de adaptação, as aves foram submetidas a quatro tratamentos, em delineamento do tipo crossover, com intervalo de 15 dias. Foram avaliadas duas associações anestésicas, midazolam $(5 \mathrm{mg} / \mathrm{kg})$ e cetamina racêmica $(15 \mathrm{mg} / \mathrm{kg})$ ou midazolam e cetamina $\mathrm{S}+(15 \mathrm{mg} / \mathrm{kg})$, administrados pela via intranasal (IN) ou pela via intramuscular (IM), obtendo-se assim os grupos midazolam e cetamina racêmica intranasal (INR), midazolam e cetamina $\mathrm{S}+$ intranasal (INS), midazolam e cetamina racêmica intramuscular (IMR) e midazolam e cetamina S+ intramuscular (IMS).

No dia do experimento, as aves foram pesadas e os anestésicos aspirados separadamente em seringas de insulina de 30 UI, sendo a cetamina racêmica diluída em água bidestilada para se obter a mesma concentração e volume da cetamina $\mathrm{S}+$. Para a administração dos fármacos, os periquitos foram contidos em decúbito dorsal, sendo que a aplicação intramuscular (IM) foi realizada nos músculos peitorais e, no caso da intranasal (IN), cada fármaco foi administrado isolado e lentamente em uma das narinas.

Após a administração dos fármacos, as aves foram posicionadas em decúbito dorsal sobre colchão térmico e então, foram avaliados, a cada cinco minutos, os tempos de latência (tempo decorrido entre a administração dos fármacos até a observação dos efeitos clínicos, caracterizado pelo decúbito e redução dos reflexos protetores), tempo de decúbito dorsal (período compreendido entre o período de latência e a primeira tentativa de levantar), tempo de anestesia (período compreendido entre o tempo de latência e o retorno dos reflexos) e tempo de recuperação (período compreendido do final do tempo de anestesia até o momento em que a ave consiguisse permanecer em pé e retomar suas atividades). O grau de sedação foi avaliado após cinco minutos da administração dos fármacos e classificado em quatro graus (S0-S3) por meio de uma escala baseada nos estudos de Valverde et al. ${ }^{(19)}$ e Kamiloglu et al. ${ }^{(1)}$, no qual: S0 foi considerada a melhor sedação, sendo que a ave permanecia com os olhos fechados, em decúbito, relaxada e sem movimentação; S1: ave com olhos abertos, em decúbito, relaxada e apresentava resposta leve a estímulos (resposta à manipulação de asa e perna); S2: ave com olhos abertos, piscando, em decúbito, pouco relaxada, com resposta a estímulos e movimentação leve; S3: ave acordada, ausência de decúbito e responsiva a estímulos.

A qualidade da recuperação foi classificada conforme a escala de Kamiloglu et al. ${ }^{(1)}$ em três graus (R1-R3), no qual R1 correspondia à ave quieta e tranquila; R2: ave com ataxia e tremores ocasionais; R3: ave com ataxia e tremores moderados.

Os resultados foram submetidos ao teste de normalidade de Kolmogorov - Smirnov e os dados paramétricos foram avaliados por análise de variância de uma via seguida por teste de Student Newman Keuls e os dados não paramétricos foram submetidos ao teste Kruskal-Wallis, ambos com $5 \%$ de significância.

\section{Resultados}

Os períodos de latência observados diferiram significativamente apenas entre INS e IMR, com tempos de 40,25 $\pm 10,55$ e 74,32 $\pm 21,77$ segundos, respectivamente. O tempo de decúbito dorsal e tempo de anestesia (Tabela 1) apresentaram diferença estatística, uma vez que os grupos INS e INR apresentaram tempos menores que dos grupos IMS e IMR. O tempo de recuperação foi significativamente maior no grupo INS comparado com o grupo IMR.

A avaliação do grau de sedação demonstrou diferença entre INR e os grupos INS e IMS (Tabela 2). Já a qualidade de recuperação não apresentou diferenças significativas entre os grupos (Tabela 2). 
Tabela 1: Médias e desvios-padrão do periodo de latência, tempo de decúbito dorsal, tempo de anestesia e tempo de recuperação de periquitos-australianos (Melopsittacus undulatus) $(\mathrm{n}=8)$ anestesiados com midazolam e cetamina $\mathrm{S}+$ ou racêmica pelas vias intranasal ou intramuscular

\begin{tabular}{ccccc}
\hline & \multicolumn{2}{c}{ Intranasal (IN) } & \multicolumn{2}{c}{ Intramuscular (IM) } \\
\hline & S+ (S) & Racêmica (R) & S+ (S) & Racêmica (R) \\
\hline Período de latência (seg) & $40,25 \pm 10,55^{\mathrm{A}}$ & $59,00 \pm 17,26$ & $49,88 \pm 14,89$ & $74,32 \pm 21,77^{\mathrm{A}}$ \\
\hline $\begin{array}{c}\text { Tempo de decúbito } \\
\text { dorsal(min) }\end{array}$ & $23,93 \pm 7,51^{\mathrm{A}, \mathrm{B}}$ & $28,68 \pm 16,13^{\mathrm{C}, \mathrm{D}}$ & $60,08 \pm 27,37^{\mathrm{A}, \mathrm{C}}$ & $74,3 \pm 21,77^{\mathrm{B}, \mathrm{D}}$ \\
\hline Tempo de anestesia (min) & $45,48 \pm 17,94^{\mathrm{A}, \mathrm{B}}$ & $39,24 \pm 15,62^{\mathrm{C}, \mathrm{D}}$ & $75,84 \pm 20,20^{\mathrm{A}, \mathrm{C}}$ & $79,4 \pm 20,73^{\mathrm{B}, \mathrm{D}}$ \\
\hline Tempo de recuperação (min) & $21,55 \pm 18,43^{\mathrm{A}}$ & $10,56 \pm 7,08$ & $15,76 \pm 9,13$ & $5,1 \pm 3,56^{\mathrm{A}}$ \\
\hline
\end{tabular}

Letras iguais na mesma linha indicam diferença estatística entre os grupos $(\mathrm{P}<0,05)$; seg: segundos; $\min$ : minutos.

Tabela 2: Porcentagens do grau de sedação e qualidade de recuperação de periquitos australianos (Melopsittacus undulatus) $(\mathrm{n}=8)$ anestesiados com cetamina $\mathrm{S}+\mathrm{e}$ cetamina racêmica, utilizando as vias intranasal ou intramuscular

\begin{tabular}{cccccc}
\hline & & \multicolumn{2}{c}{ Intranasal (IN) } & \multicolumn{2}{c}{ Intramuscular (IM) } \\
\cline { 2 - 6 } & & S+ (S) & Racêmica (R) & S+ (S) & $\begin{array}{c}\text { Racêmica } \\
\text { (R) }\end{array}$ \\
\hline Grau de sedação & S0 & $8(100 \%)^{\mathrm{A}}$ & $3(37,5 \%)^{\mathrm{A}, \mathrm{B}}$ & $8(100 \%)^{\mathrm{B}}$ & $7(87,5 \%)$ \\
\cline { 2 - 6 } & S1 & $0(0 \%)$ & $4(50 \%)$ & $0(0 \%)$ & $1(12,5 \%)$ \\
\cline { 2 - 6 } & S2 & $0(0 \%)$ & $1(12,5 \%)$ & $0(0 \%)$ & $0(0 \%)$ \\
\cline { 2 - 6 } & S3 & $0(0 \%)$ & $0(0 \%)$ & $0(0 \%)$ & $0(0 \%)$ \\
\hline Qualidade de & R1 & $7(87,5 \%)$ & $6(75 \%)$ & $4(50 \%)$ & $8(100 \%)$ \\
\cline { 2 - 6 } recuperação & R2 & $1(12,5 \%)$ & $2(25 \%)$ & $3(37,5 \%)$ & $0(0 \%)$ \\
\cline { 2 - 6 } & R3 & $0(0 \%)$ & $0(0 \%)$ & $1(12,5 \%)$ & $0(0 \%)$ \\
\hline
\end{tabular}

S0: ave com os olhos fechados, em decúbito, relaxado e sem movimentação; S1: ave com olhos abertos, em decúbito, relaxada e resposta leve a estímulos; $\mathrm{S} 2$ : ave com olhos abertos, piscando, em decúbito, relaxada, com resposta a estímulos e movimentação leve; S3: ave acordada, ausência de decúbito e responsiva a estímulos. R1 corresponde à ave quieta e tranquila; R2: ave com ataxia e tremores ocasionais; R3: ave com ataxia e tremores moderados. Letras iguais na mesma linha indicam diferença estatistica entre os grupos $(\mathrm{P}<0,05)$.

\section{Discussão}

As doses dos fármacos empregados neste estudo encontram-se dentro dos valores descritos na literatura para a anestesia de aves e se mostraram eficientes para a anestesia de curta duração em periquitos. Em virtude da grande variedade de espécies, são descritas diferentes doses para uso pela via intranasal em aves. Vesal \& Eskandari ${ }^{(6)}$ descreveram o uso de $3,36 \mathrm{mg} / \mathrm{kg}$ de midazolam associado a 40 a $50 \mathrm{mg} /$ $\mathrm{kg}$ de cetamina em periquitos. Em canários, Vesal \& Zare ${ }^{(3)}$ relataram doses entre 12,5 e 15,6 mg/kg de midazolam, Beier et al. ${ }^{(14)}$ utilizaram $20 \mathrm{mg} / \mathrm{kg}$ de cetamina associada a 3,5 $\mathrm{mg} / \mathrm{kg}$ de midazolam em pombas, Bitencourt et al. ${ }^{(15)}$ utilizaram doses de $15 \mathrm{mg} / \mathrm{kg}$ de cetamina e $1 \mathrm{mg} / \mathrm{kg}$ de midazolam. Já Bigham \& Moghaddam ${ }^{(13)}$ recomendaram a dose de $13 \mathrm{mg} / \mathrm{kg}$ de midazolam em verdilhões e, segundo 
estes autores, a anestesia pela via intranasal proporcionou sedação de boa qualidade com rápida recuperação. Embora no presente estudo tenha-se empregado uma dose mais baixa de midazolam $(5 \mathrm{mg} / \mathrm{kg})$, foram observados resultados semelhantes.

A diferença observada entre os grupos INS e IMR para o tempo de latência pode ser atribuída à diferença entre a via de administração e aos fármacos, sendo que a via IM requer maior tempo para que ocorra a absorção do anestésico, quando comparado à via intranasal. Além disso, a cetamina racêmica apresenta menor potência anestésica quando comparada à cetamina $S+{ }^{(16,20)}$, justificando $o$ maior tempo de latência no grupo IMR.

A via intranasal apresenta rápida absorção ${ }^{(21)}$, justificando diferença observada nos tempos de decúbito e tempo de anestesia, sendo que os grupos INS e INR apresentaram tempos menores que nos grupos IMS e IMR. Bigham \& Moghaddam ${ }^{(13)}$ relataram que a via intranasal proporciona rápida biotransformação, resultando em efeitos de curta duração em aves. Resultados semelhantes foram obtidos por Beier et al. ${ }^{(14)} \mathrm{em}$ pombas e por Bitencourt et al. ${ }^{(15)} \mathrm{em}$ papagaios e, em ambos os casos, os tempos de anestesia foram menores nas aves anestesiadas pela via IN. O tempo total de anestesia pela via IN foi próximo ao relatado por Vesal \& Zare ${ }^{(3)}$ em canários com o uso de $13,6 \mathrm{mg} / \mathrm{kg}$ de midazolam, demonstrando que a redução da dose deste fármaco associado ao uso da cetamina não gera alteração significativa no tempo de anestesia.

O maior tempo de recuperação observado nas aves em que se administrou cetamina S+ pode sugerir que esta formulação apresente maior potência anestésica em periquitos, assim como descrito em $\operatorname{ratos}^{(16)}$ e cães ${ }^{(17)}$. Avaliando o tempo de recuperação de INS e IMR, a diferença observada pode ser explicada pela maior potência da cetamina $\mathrm{S}+$ que, mesmo apresentando maior biotransformação pela via $\mathrm{IN}^{(21)}$, pode ter resultado em concentrações sub-anestésicas de cetamina que não proporcionaram anestesia porém acarretaram maior tempo de recuperação, sendo necessários mais estudos com cetamina $\mathrm{S}+$ em periquitos para confirmar esta teoria.

Os resultados de tempo de recuperação divergem dos observados nos estudos de Beier et al. ${ }^{(14)} \mathrm{e}$ Bitencourt et al. ${ }^{(15)}$, os quais encontraram menor tempo de recuperação quando utilizaram a via IN em pombas e papagaios comparada à via IM. Este resultado também era esperado, uma vez que a via IN apresenta maior velocidade de metabolização e, portanto, efeitos hipnóticos e de recuperação mais rápidos ${ }^{(13)}$, o fato de obtermos maiores tempos de recuperação em IN pode ser atribuído às doses utilizadas e à diferença de metabolismo entre as espécies.

O melhor grau de sedação observado nas aves em que se utilizou a cetamina S+ é justificado pela maior potência desta formulação ${ }^{(16,17)}$. A qualidade de recuperação não diferiu entre os grupos, demonstrando que independente da via de administração e da formulação de cetamina, as doses propostas promoveram recuperação calma e tranquila,em $78,12 \%$ dos periquitos.

Dentre os efeitos adversos observados, destacaram-se o padrão respiratório abdominal (100\%) e cianose (25\%) em INR, ao passo que em INS não se observou nenhuma complicação anestésica. Estes resultados sugerem que, apesar da maior potência anestésica da cetamina $S+{ }^{(16)}$, esta causa menor depressão respiratória e, assim como observado por Beier et al. ${ }^{(14)}$, mostrou-se livre de efeitos adversos quando utilizada pela via IN. Observou-se que 47,6\% dos periquitos espirraram após a administração intranasal; entretanto, acredita-se que o espirro não causou nenhum prejuízo significativo na absorção dos fármacos, uma vez que ocorreram com média de um minuto após a administração e todas as aves apresentaram o efeito anestésico esperado. Não foi observado nenhum efeito adverso nas aves dos grupos IMR e IMS.

Neste estudo, foi preconizado a utilização de materiais de baixo custo e fácil aquisição para a rotina do médico veterinário, por este motivo, foi utilizada seringa de insulina de 30 UI para a administração dos fármacos, assim como descrito por Mans et al. ${ }^{(4)}$, ao contrário dos estudos de Vesal \& Zare ${ }^{(3)}$, Vesal \& Eskandari( ${ }^{(6)}$ e Beier, et al. ${ }^{(14)}$, que utilizaram pipetas do tipo eppendorf. A administração IN com seringa de insulina acarretou em lesão puntiforme na narina com sangramento leve em 7 periquitos $(16,6 \%)$, de um total de 42 procedimentos de sedação intranasal realizados, incluindo o estudo piloto. Em todos os casos, o sangramento foi de rápida e fácil hemostasia, não sendo necessário realizar nenhum procedimento hemostático. Em ambos os grupos foram observados movimentos de asa e pernas logo após a administração dos fármacos, porém não foi possível diferir se houve diferença de reação entre os grupos ou se a reação foi decorrente apenas da contenção física.

Este estudo demonstrou que a via intranasal propiciou bom grau de sedação, com recuperação rápida 
e de boa qualidade em periquitos, mostrando-se uma via de administração eficaz para realização de procedimentos rápidos e simples, assim como relatado em pombas-rola ${ }^{(14)}$, periquitos-de-colar ${ }^{(6)}$, canários $^{(3)}$ e papagaios ${ }^{(4,15)}$.

\section{Conclusão}

A administração da associação de midazolam e cetamina racêmica ou cetamina S+ pela via intranasal ou intramuscular nas doses propostas mostrou-se eficaz e viável para a anestesia de periquitos australianos (Melopsittacus undulatus). As associações propostas podem ser utilizadas em procedimentos de curta duração e não invasivos. Quanto às vias de administração, provou-se que a via intranasal apresenta menores tempos de decúbito e anestesia que a via intramuscular, sendo indicada para procedimentos rápidos em que se deseja a rápida recuperação do paciente.

\section{Referências}

1. Kamiloglu A, Atalan G, Kamiloglu NN. Comparison of intraosseous and intramuscular drug administration for induction of anaesthesia in domestic pigeons. Research in Veterinary Science. 2008;85(1):171-175

2. Machin KL. Waterfowl anesthesia. Semi in Avi and Exo Pet Med. 2004 out;13(4):206-12. DOI http://dx.doi. org/10.1053/j.saep.2004.04.006.

3. Vesal N, Zare P. Clinical evaluation of intranasal benzodiazepines, $\propto_{2}$-agonista and their antagonists in canaries. Veterinarian Anes and Analgesia. 2006;33:143-148

4. Mans C, Guzman DS, Lahner LL, Paul-murphy J, Sladky KK. Sedation and physiologic response to manual restraint after intranasal administration of midazolam in hispaniolan Amazon parrots (Amazonaventralis). Jour of Avi Med and Surg. 2012; 26(3):130-139

5. Forbes NA. Avian anesthesia. Vet Quart. 1998;20(1):65-6. DOI http://dx.doi.org/10.1080/01652176.1998. 10807418.

6. Vesal N, Eskandari MH. Sedative effects of midazolam and xylazine with or without ketamine and detomidine alone following intranasal administration in Ring-necked Parakeets. Jour of the Amer Vet Med Assoc. 2006 fev 1; 228:383-388. DOI http://dx.doi.org/10.2460/javma.228.3.383

7. Hawkins MG, Pascoe PJ, Cagebirds In West, G, Heard D, Caulkett N. Zoo animal and wildlife immobilization and anesthesia. Black Pub. 2007:283-85. DOI http://dx.doi.org/10.1638/1042-7260-40.4.815

8. Gunkel C, Lafortune M. Current Techniques in Avian Anesthesia. Semi in Avi and Exo Pet Med. 2005 out;14(4):263-76. DOI http://dx.doi.org/10.1053/j.saep.2005.09.006.

9. Murphy JP, Fialkowski J. Injectable anesthesia and analgesia of birds. In: Gleed RD, Ludders JW. Recent Advance in Veterinary Anesthesia and Analgesia, Companion animals. New York: International Veterinary Information Service. Ithaca NY (www.ivis.org), 2001. 14p.

10. Mama MR, Phillips LG, Pascoe PJ. Use of propofol for induction and maintenance of anesthesia in a barn owl (Tyto alba) undergoing tracheal resection. Jour of Zoo Wild Med. 1996;27(3):397-401.

11. Curro TG. Anesthesia of pet birds. Sem in Avi and Exo Pet Med.1998 jan;7(1):10-21 DOI http:// dx.doi. org/10.1016/S1055-937X(98)80053-4.

12. Machin KL, Caulkett NA. Investigation of injectable anesthetic agents in Mallard Ducks (Anas platyrhynchos): a descriptive study. Jour of Avi Med and Surg.1998;12:255-62.

13. Bigham AS, Moghaddam AKZ. Finch (Taeneopygiaguttata) sedation with intranasal administration of diazepam, midazolam or xylazine. J. vet. Pharmacol. Therap. 2009 maio 9;36(1):1-3. DOI http:// dx.doi. org/10.1111/j.1365-2885.2009.01102.x. 
14. Beier SL, Rosa AC, Oleskovicz N, Mattoso CRS, Moraes NA. Efeitos anestsicos da administração intranasal ou intramuscularde cetamina $\mathrm{S}+$ e midazolam em pomba-rola (Streptotelia $s p$.). Pesq Vet Bras.2013;33(4):517-22. DOI http://dx.doi.org/10.1590/S0100-736X2013000400016.

15. Bitencourt EH, Padilha VS, Lima MPA, Beier SL, Moraes AN. Efeitos sedativos da associação de cetamina e midazolam administrados pela via intranasal ou intramuscular em papagaio (Amazona aestiva e Amazona vinacea). Pesq Vet Bras. 2013;33(9):1125-9. DOI http://dx.doi.org/10.1590/S0100-736X2013000900013.

16. Marietta MP, Way WL, Casta N, Trevor AJ. On the pharmacology of ketamine enantiomorphs in the rat. Jour of Pharm and Expl Therap.1997;202:257-263.

17. Duque JC, Oleskovicz N, Guirro ECBP, Valadão CAA, Soares VE. Relative potency of ketamine and $\mathrm{S}(+)$-ketamine in dogs. J. vet Pharmacol. Therap. 2008;31:344-8. DOI http://dx.doi.org/10.1111/ j.1365-2885.2008.00965.x.

18. Lauretti GR, Lima IC, Buscatti RY. Avaliação clínica dos efeitos hemodinâmicos, analgésicos, psicodélicos e do bloqueio neuromuscular da cetamina racêmica e do seu (+)-isômero. Rev Bras Anes. 2000;50:357-62.

19. Valverde A, Bienzle D, Smith DA, Dyson DH,Valliant AE.Intraosseous cannulation and drug administration for induction of anesthesia in chickens. Vet Surg. 1993;22(3):240-4. DOI http://dx.doi.org/10.1111/ j.1532-950X.1993.tb00392.x.

20. Sinner B, Graf BM. Ketamine. In: Schüttler J, Schwilden H. Handobook of Experimental Pharmacology: Modern Anesthetics. Berlin; Springer, 2008. p.313-333.

21. Bjorkman S, Rigemar G, Idvall J. Pharmacokinetics of midazolam given as an intranasal spray to adult surgical patients. Brit Jour Anaes. 1997;79:575-580. 